\title{
Desempenho térmico-acústico-mecânico de um compósito de matriz cimentícia leve reforçado com casca de arroz
}

\author{
Thermal-acoustic-mechanical performance \\ of a lightweight cementitious matrix \\ composite reinforced with rice husk
}

\author{
Eduardo Pachla ${ }^{1}$, Débora Silva ${ }^{1}$, Pedro Jucá ${ }^{1}$ \\ Wang Chong ${ }^{1}$, Ederli Marangon ${ }^{1}$
}

\footnotetext{
${ }^{1}$ Universidade Federal do Pampa - UNIPAMPA - Alegrete, Rio Grande do Sul, Brasil. e-mail: eduardo.pachla@gmail.com, deborabretas13@gmail.com, pedrohojuca@gmail.com, wangchong@unipampa.edu.br ederlimarangon@gmail.com
}

\begin{abstract}
RESUMO
Visando desenvolver um novo produto com bom desempenho térmico-acústico-mecânico para servir como alternativa aos materiais comumente utilizados para vedação, foi produzido um compósito a partir de uma matriz cimentícia leve e casca de arroz. A matriz cimentícia consistindo em uma pasta de cimento com ar incorporado, que é intitulada por vários pesquisadores como concreto celular, tem como características principais sua reduzida massa específica e o grande número de pequenos poros não interligados. Por sua vez, a casca de arroz é uma fibra vegetal oriunda da benfeitoria do arroz abundante no estado do Rio Grande do Sul, que é o maior produtor nacional do grão, e que não possui atualmente uma destinação adequada. O concreto celular e o compósito (concreto celular mais casca de arroz) foram testados quanto à resistência mecânica à compressão axial simples e quanto aos desempenhos acústico e térmico. Em relação à resistência à compressão, apesar de constatar-se que a casca de arroz influenciou negativamente no produto, ambos os materiais atingiram respectivamente as resistências mínimas de 1,20 MPa e 1,50 MPa estipuladas pelas NBR 13.438 e NBR 15.270, entretanto, somente a matriz atendeu aos 3,00 MPa exigidos pela NBR 6136. Na análise acústica, o compósito obteve um melhor desempenho quanto a absorção acústica, por outro lado, quanto ao isolamento acústico os materiais não apresentaram valores estatisticamente diferentes, impossibilitando afirmar qual produto é superior. No que concerne a condutividade térmica, o compósito com casca de arroz apresentou desempenho inferior à matriz, fato atribuído à elevada quantidade de matéria orgânica presente nas fibras.
\end{abstract}

Palavras-chave: concreto celular, casca de arroz, desempenho acústico, condutividade térmica, resistência mecânica.

\begin{abstract}
Aiming to develop a new product with good thermal-acoustic-mechanical performance to serve as an alternative to the materials commonly used for sealing, a composite was fabricated from a light cementitious matrix and rice husk. The cement matrix consisting in a cement paste with incorporated air, titled by several researchers as cellular concrete, presents main characteristics such as low specific mass and the large number of non-interconnected small pores. In turn, the rice husk is a vegetal fiber derived from the residue of rice production abundant in the Rio Grande do Sul state, the largest national producer of the grain. The cellular concrete and the composite (cellular concrete plus rice husk) were tested for mechanical resistance by single axial compression and by acoustic and thermal performance. Regarding the compressive strength, although the rice husk had a negative influence on the product, the both materials reached respectively the minimum strengths of $1.20 \mathrm{MPa}$ and $1.50 \mathrm{MPa}$ stipulated by NBR 13.438 and NBR 15.270, however, only the matrix (cellular concrete) hit the $3.00 \mathrm{MPa}$ required by the NBR 6136. For the acoustic test, the composite obtained a better performance in acoustic absorption; on the other hand, concerned with the acoustic insulation the two materials did not present statistically difference, thus, one is impossible to affirm which one is better. About, the thermal conductivity, the composite with rice husk presented inferior performance to the matrix, which would be attributed to the high organic matter content present in the fibers.
\end{abstract}

Keywords: cellular concrete, rice husk, acoustic performance, thermal conductivity, mechanical strength. 


\section{INTRODUÇÃO}

A tragédia da boate Kiss, ocorrida em 2013 na cidade de Santa Maria - RS, causou a perda de 242 vidas devido a um incêndio que teve como protagonista a espuma de isolamento acústico de poliuretano. $\mathrm{Na}$ circunstância a combustão da espuma liberou gases tóxicos como monóxido de carbono, dióxido de carbono e gás cianídrico, culminando na morte por asfixia de pelo menos 234 das vítimas da tragédia $[1,2]$. O ocorrido alavancou uma série de questionamentos pertinentes no que diz respeito à legislação, fiscalização e ao uso de materiais sintéticos que são empregues para conferir um maior conforto ambiental.

Conjuntamente à isso, o desenvolvimento de novos materiais que possam cumprir a necessidade de isolar ambientes, não só acusticamente, mas também termicamente, passou a ser requisitado pela norma de desempenho NBR 15.575 [3]. Cenário que paralelamente à tragédia destacou a importância do desenvolvimento de materiais mais tecnológicos, sem o uso de adições sintéticas que possam liberar gases tóxicos em caso de combustão ou contato com outros produtos químicos.

Nesse sentido, o uso de materiais compósitos surge como uma opção viável para a elaboração de produtos que possam oferecer maior desempenho térmico-acústico, uma vez que sua principal característica é a melhoria das propriedades finais em comparação às de seus constituintes separadamente [4]. Ademais, baseado no fato de que concretos, argamassas e pastas de cimento com elevada porosidade apresentam bons resultados frente ao desempenho térmico-acústico [5], definiu-se como matriz do compósito uma pasta de cimento com ar incorporado, material este denominado de concreto celular por diversos autores [ $6,7,8]$.

Além da quantidade de poros ser um fator preponderante para a capacidade do material absorver som e servir como isolante térmico, é sabido que a dimensão dos poros também tem relação com o desempenho térmico-acústico [ 9, 10,11]. Uma vez que o Rio Grande do Sul é o maior produtor de arroz do Brasil [12], e a casca de arroz (CA) é um resíduo agrícola com aproveitamento limitado, tendo como atual destino a queima para geração de energia elétrica e secagem de cereais [13], foi estabelecida a incorporação da mesma ao concreto celular a fim de aumentar a dimensão dos poros originais da matriz.

Os benefícios ambientais promovidos pela adição de fibras vegetais, que em grande parte são resíduos que possuem destino inadequado, não são restringidos a um destino ecologicamente correto, concomitantemente a isto, os mesmos podem trazer a redução do consumo de energia, pois a incorporação de alguns tipos de fibras naturais ou sintéticas em compósitos de matriz cimentícia diminui a condutividade térmica do produto em consequência do aumento da porosidade, o que gera uma redução na utilização de equipamentos mecânicos para refrigeração das edificações [11, 14].

Em face destas considerações, este trabalho propõe a incorporação de casca de arroz (CA) em uma matriz cimentícia leve para conceber um destino sustentável à este resíduo agrícola e aperfeiçoar o desempenho térmico-acústico da matriz. Além disso, busca-se alcançar a resistência mecânica mínima requisitada por normas brasileiras, para viabilizar a utilização dos compósitos na construção civil.

\section{MATERIAIS E MÉTODOS}

Para a elaboração da matriz cimentícia e do compósito contendo casca de arroz (CA) foi utilizado Cimento Portland de Alta Resistência Inicial Resistente à Sulfatos. Como na cidade de Alegrete - Rio Grande do Sul, onde foi realizada a pesquisa, não há disponibilidade do CPV-ARI, optou-se pelo uso do CPV-ARI-RS uma vez que o mesmo possuí menor proporção de adições em relação aos demais tipos de Cimento Portland. Além disso, o uso de CPV-ARI obtido de regiões distantes implicaria em maior dificuldade na implementação dos produtos desenvolvidos na construção civil regional, uma vez que a indústria e o comércio local não possuiriam a matéria prima necessária para produção dos compósitos em larga escala.

A casca de arroz não passou por nenhum tipo de tratamento químico ou físico e foi inserida na mistura de forma totalmente natural conforme apresentado pela Figura 1a. Fernandes et al. [15] caracterizaram a casca de arroz proveniente de Alegrete. Nessa caracterização a massa específica encontrada foi $1,41 \mathrm{~g} / \mathrm{cm}^{3}$, valor muito semelhante com outras bibliografias estudadas [16, 17]. Além da massa específica Fernandes et al. [15] determinaram que a casca apresenta granulometria homogênea gaussiana e aproximadamente $93 \%$ de seu conteúdo ficou retido entre as peneiras com abertura de 0,85 e 1,70 mm. Ademais, segundo Ferreira [18] a CA é composta por 82,70\% de componentes orgânicos (43,50\% de celulose, $22,00 \%$ de hemicelulose e $17,20 \%$ de lignina) e $17,30 \%$ de outros componentes inorgânicos.

$\mathrm{O}$ aditivo químico utilizado na produção da espuma (Figura 1b) foi o incorporador de ar e plastificante produzido através de uma solução aquosa de sal de sódio e ácido sulfônico denominado de ECOFOAM-AIR produzido pela ECOPORE. A massa específica do aditivo é de 1,01 g/cm², $\mathrm{pH} 4,0$ e 
teor de sólidos de 5,7\%. O aditivo foi misturado com água na proporção de $1 \mathrm{~kg}$ de aditivo para cada 40 $\mathrm{kg}$ de água. A espuma foi obtida a partir do incorporador de ar ECOFOAMER-100W-10BP, o qual é responsável por injetar ar na mistura aquosa gerando uma espuma com densidade aproximada de $75 \mathrm{~g} / 1$.

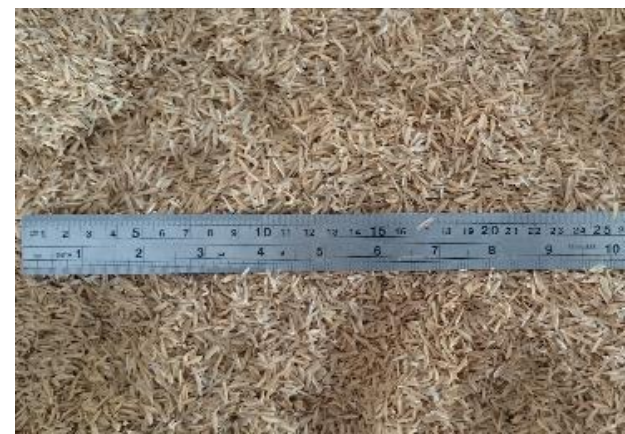

(a)

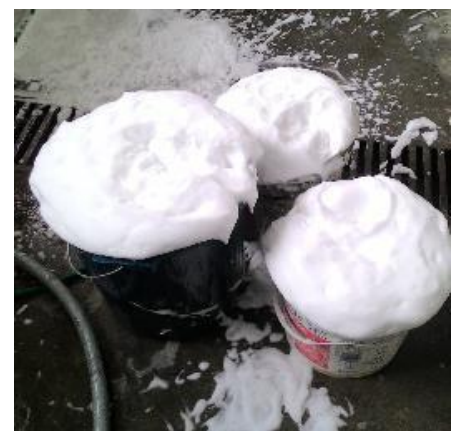

(b)

Figura 1: Casca de arroz (a) e espuma pré-formada (b).

O tipo de concreto celular produzido se enquadra no grupo dos concretos celulares aerados por agente espumígeno, e para tal utiliza-se a espuma pré-formada (Figura 1b). Os materiais são provenientes da combinação de uma pasta de cimento com água e espuma nas proporções apresentadas na Tabela 1.

Tabela 1: Traços massa combinado com volume.

\begin{tabular}{c|c|c}
\hline MATERIAIS & MATRIZ CIMENTÍCIA LEVE & COMPÓSITO COM CASCA DE ARROZ \\
\hline Cimento $(\mathrm{kg})$ & 1,00 & 1,00 \\
\hline Casca de Arroz (1) & 0,00 & 0,35 \\
\hline Água na mistura (1) & 0,40 & 0,40 \\
\hline Espuma pré-formada (1) & 2,80 & 2,80 \\
\hline Água na Espuma (1) & 0,10 & 0,10 \\
\hline Fator a/c & 0,50 & 0,50 \\
\hline
\end{tabular}

Para cada traço foram moldados três corpos-de-prova (CP's) cilíndricos de $10 \mathrm{~cm}$ de diâmetro por $20 \mathrm{~cm}$ de altura para o ensaio de resistência à compressão axial simples, três CP's em formato de placas de $25 \mathrm{~cm} \times 25 \mathrm{~cm}$ e $5 \mathrm{~cm}$ de espessura para o ensaio de condutividade térmica. Para os ensaios de desempenho acústico foram utilizados três CP's de $10 \mathrm{~cm}$ de diâmetro por $5 \mathrm{~cm}$ de espessura para frequência de $50 \mathrm{~Hz}$ à $1.600 \mathrm{~Hz}$ e três CP's de $2,90 \mathrm{~cm}$ de diâmetro por $5 \mathrm{~cm}$ de espessura para a faixa de frequência de $500 \mathrm{~Hz}$ à $6.300 \mathrm{~Hz}$. Salienta-se que a dimensão dos corpos-de-prova é limitada em relação à dimensão da seção transversal do tubo de impedância, sendo esta a maior desvantagem do método, visto que tal dimensão acaba delimitando a maior frequência de ensaio [19].

O procedimento de moldagem dos corpos de prova de concreto celular foi baseado na NBR 5738 [20], no que se refere à aparelhagem, ao rasamento, ao manuseio e ao transporte. Cabe salientar que não foi realizado nenhum tipo de adensamento, ou seja, o concreto foi simplesmente vertido dentro das fôrmas a fim de evitar o rompimento das bolhas de ar da espuma, o que alteraria as características microestruturais do produto final.

Após a desmoldagem dos corpos-de-prova, os mesmos foram mantidos em temperatura ambiente até a idade de 27 dias. Nessa idade, os CP's tiveram as superfícies niveladas com serra elétrica na presença de água. Para uniformizar a umidade superficial incrementada às amostras após o nivelamento superficial elas foram colocadas em estufa sob temperatura de $65^{\circ} \mathrm{C}$ durante 24 horas. Os ensaios de compressão axial foram realizados aos 28 dias de cura e em um período varíavel de 28 à 30 dias de cura foram realizados os ensaios para a determinação das propriedades acústicas e térmicas. A cura em temperatura ambiente ocorreu com o intuito de criar maior similaridade com o concreto produzido 'in loco', conforme indicado pela NBR 5738 [20] para o caso de CP's moldados para verificar as condições de proteção e cura do concreto.

Os ensaios de resistência à compressão axial foram realizados em uma máquina de ensaio universal de capacidade de $200 \mathrm{kN}$ com velocidade de $0,50 \mathrm{~mm} / \mathrm{min}$. Os ensaios de absorção e isolamento acústico foram realizados em um tubo de impedância, com a emissão de ruído branco em conformidade com a norma ISO 10534-1 [21]. A condutividade térmica foi determinada pelo método do prato quente e 
os cálculos foram realizados utilizando-se a Lei de Fourier, conforme indicado pela norma ASTM C518 [22].

As análises estatísticas dos resultados encontrados para resistência à compressão axial, absorção e isolamento acústico e condutividade térmica foram realizadas pela comparação entre as faixas de dispersão obtidas através do desvio padrão (D.P.) e pela amplitude dos desvios em relação ao valor médio, a qual foi verificada através do coeficiente de variação (C.V.).

\section{RESULTADOS E DISCUSSÃO}

\subsection{Resistência à compressão axial}

Os resultados dos ensaios de resistência à compressão axial aos 28 dias de cura constam na Tabela 2.

Tabela 2: Resistências à compressão axial (valor médio).

\begin{tabular}{c|c|c|c}
\hline MATERIAL & $\begin{array}{c}\text { RESISTÉNCIA À COMPRESSÃO } \\
(\mathbf{M P a})\end{array}$ & $\begin{array}{c}\text { DESVIO PADRÃO } \\
\text { (MPa) }\end{array}$ & $\begin{array}{c}\text { C. V. } \\
\text { (\%) }\end{array}$ \\
\hline Matriz cimentícia leve & 3,16 & 0,22 & 6,90 \\
\hline Compósito com casca de arroz & 2,85 & 0,07 & 2,46 \\
\hline
\end{tabular}

A Figura 2 ilustra de forma gráfica os dados expostos na Tabela 2, assim facilitando o entendimento e possibilitando verificar a confiabilidade dos mesmos através das barras de dispersão. É importante salientar que o objetivo era atingir às resistências à compressão axial mínima estipulada por algumas normas brasileiras para materiais de vedação. Nesse sentido, ambas as composições atenderam às exigências da NBR 13.438 e da NBR 15.270 [23, 24], que solicitam respectivamente $1,20 \mathrm{MPa} e$ 1,50 MPa. Entretanto, apenas a matriz cimentícia atendeu ao valor mínimo de 3,00 MPa exigido pela NBR 6136 [25].

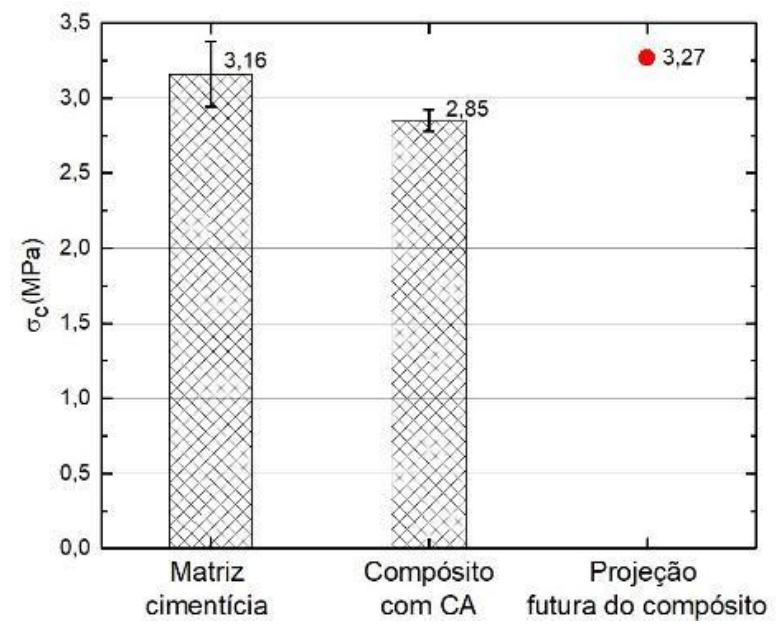

Figura 2: Resistência à compressão axial simples.

De acordo com os resultados ilustrados na Figura 2 observa-se que a casca de arroz influenciou negativamente a resistência mecânica à compressão axial, uma vez que o compósito com casca de arroz apresentou resultado inferior à matriz. Isto provavelmente ocorreu em razão do aumento da dimensão dos poros provenientes da adição da CA. Analisando as faixas de dispersão dos materiais verifica-se que elas não estão sobrepostas, o que indica que os resultados são estatisticamente diferentes.

A incorporação da casca de arroz ao concreto celular foi motivada pela presença da sílica que a mesma possui em seu estado natural [26]. A comprovação de que a sílica da casca reage com o hidróxido de cálcio do cimento originando reação pozolânica foi feita por Jauberthie et al. [27]. Em seu estudo, a matriz cimentícia combinada com casca apresentou um aumento na resistência à compressão após um ano de cura em câmara umida com 95\% de umidade relativa. Pachla [28] concluiu que a resistência à compressão axial do compósito de cimento com casca de arroz aumentou quando realizados ciclos de molhagem e secagem em um perídodo de 91 dias devido à reação da sílica presente na casca de arroz com o hidróxido de cálcio. Desta forma, espera-se que o compósito apresente resistência à compressão superior à matriz original com o avanço da idade. Essa perspectiva é ilustrada através de uma projeção futura (Figura 2) com base nos valores encontrados por Jauberthie et al. e Pachla [27, 28]. 


\subsection{Absorção acústica}

Os resultados de alguns pontos da frequência aferida nos ensaios de absorção acústica constam na Tabela 3. No total foram aferidos 22 pontos compreendidos na frequência de $50 \mathrm{~Hz}$ à $6.300 \mathrm{~Hz}$. Os valores não apresentados na Tabela 3 são ilustrados gráficamente na Figura 3. As linhas tracejadas em torno dos valores médios representam as faixas de dispersão dos resultados.

Tabela 3: Coeficiente de absorção acústica ( $\alpha$ ) para frequência de $50 \mathrm{~Hz}$ à $6300 \mathrm{~Hz}$.

\begin{tabular}{c|c|c|c|c|c|c}
\hline \multirow{2}{*}{ FREQUÊNCIA (Hz) } & \multicolumn{3}{|c|}{ MATRIZ CIMENTíCIA } & \multicolumn{2}{c}{ COMPÓSITO COM CASCA DE ARROZ } \\
\cline { 2 - 7 } & $\mathbf{\alpha}$ & D. P. & C.V. (\%) & a & D. P. & C.V. (\%) \\
\hline 50 & 0,0010 & 0,0000 & 0,00 & 0,0263 & 0,0067 & 25,28 \\
\hline 125 & 0,0027 & 0,0012 & 43,30 & 0,0357 & 0,0284 & 79,52 \\
\hline 250 & 0,0043 & 0,0015 & 35,25 & 0,0523 & 0,0272 & 51,89 \\
\hline 500 & 0,0090 & 0,0020 & 22,22 & 0,0567 & 0,0236 & 41,70 \\
\hline 1000 & 0,0120 & 0,0010 & 8,33 & 0,0493 & 0,0144 & 29,12 \\
\hline 2000 & 0,0240 & 0,0046 & 19,09 & 0,0573 & 0,0135 & 23,55 \\
\hline 4000 & 0,0267 & 0,0051 & 19,24 & 0,0783 & 0,0290 & 37,05 \\
\hline 6300 & 0,0330 & 0,0087 & 26,42 & 0,0840 & 0,0153 & 18,02 \\
\hline
\end{tabular}

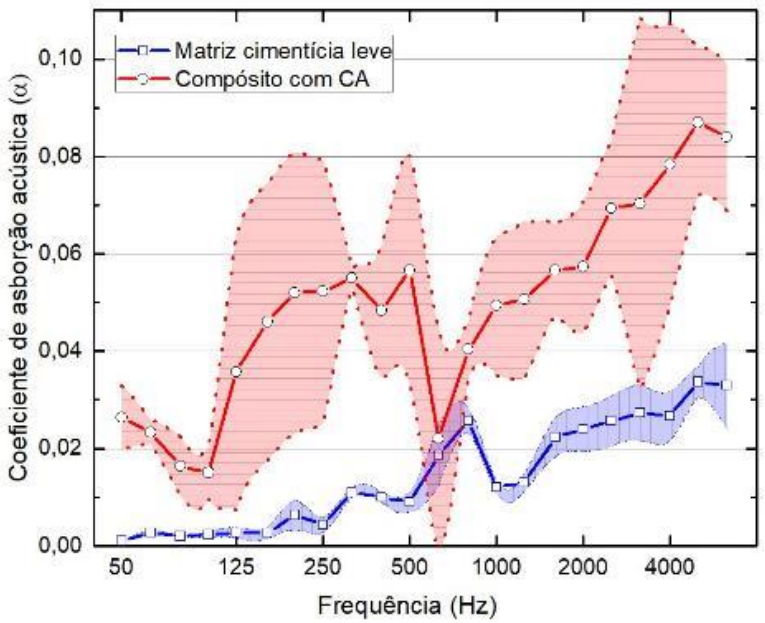

Figura 3: Curvas de absorção acústica dos materiais.

Observa-se que o compósito apresentou absorção acústica superior na maior parte da faixa de frequência analisada. Além disso, dos 22 pontos analisados apenas em um houve sobreposição das faixas de dispersão, o que indica que há diferença estatisticamente significativa entre os valores médios. Segundo [29, 30] a melhora do desempenho ocorre em virtude do aumento da dimensão dos poros, fato esse observado após a adição da casca de arroz (CA) na matriz (Figura 4).

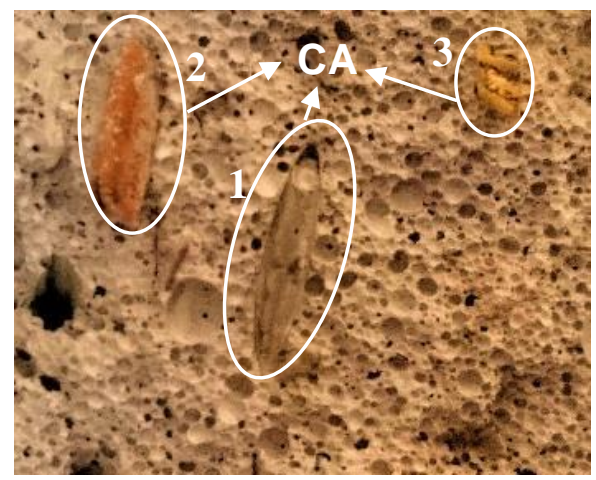

Figura 4: Ampliação da estrutura interna do compósito com casca de arroz. 
Através dos detalhes indicados na Figura 4 verifica-se que a dimensão dos poros criados pela CA (detalhe 1) é superior às dimensões dos poros originados pela espuma incorporadora de ar. Além disso, os poros originados pela CA possuem formatos irregulares e aleatórios, o que aumenta a tortuosidade do material e incrementa a absorção acústica [31,32]. No detalhe 2 nota-se que o poro gerado pela CA é envolvido pela fibra, ou seja, o poro originado pela CA é o volume ocupado pelo grão de arroz antes de sua benfeitoria. $\mathrm{O}$ detalhe 3 representa uma fibra rompida e, neste caso, o aumento da dimensão do poro criado ficará mais nítido após a decomposição da matéria orgânica, quando a CA reduzirá de volume [28].

\subsection{Isolamento acústico}

Os resultados médios dos ensaios de isolamento acústico constam na Tabela 4. A faixa de frequência analisada foi de $50 \mathrm{~Hz}$ à $6.300 \mathrm{~Hz}$, com todos os pontos ilustrados na Figura 5.

De maneira semelhante à [30, 31 e 33] a habilidade do material de isolar o som não foi reduzida pela alteração do volume e da dimensão dos poros. Esse fator pode ser verificado, pois não é possível determinar qual material obteve melhor isolamento acústico, uma vez que analisando as faixas de dispersão (ilustradas na Figura 5 com linhas pontilhadas em torno da média) pode-se concluir que na maior parte da faixa de frequência há sobreposição de dados, indicando que não ocorreu diferença estatisticamente significativa entre os resultados obtidos.

Tabela 4: Isolamento acústico para frequência de $50 \mathrm{~Hz}$ a $6.300 \mathrm{~Hz}$.

\begin{tabular}{c|c|c|c|c|c|c}
\hline \multirow{2}{*}{ FREQUÊNCIA (Hz) } & \multicolumn{3}{|c|}{ MATRIZ CIMENTÍCIA } & \multicolumn{3}{c}{ COMPÓSITO COM CASCA DE ARROZ } \\
\cline { 2 - 7 } & $\begin{array}{c}\text { ISOLAMENTO } \\
\text { (dB) }\end{array}$ & $\begin{array}{c}\text { D. P. } \\
\text { (dB) }\end{array}$ & C.V. (\%) & $\begin{array}{c}\text { ISOLAMENTO } \\
\text { (dB) }\end{array}$ & $\begin{array}{c}\text { D. P. } \\
\text { (dB) }\end{array}$ & C.V. (\%) \\
\hline 50 & 11,70 & 0,74 & 6,29 & 16,00 & 1,49 & 9,28 \\
\hline 125 & 20,96 & 1,18 & 5,62 & 22,71 & 1,43 & 6,31 \\
\hline 250 & 17,72 & 2,04 & 11,52 & 18,02 & 1,43 & 7,93 \\
\hline 500 & 33,27 & 2,90 & 8,72 & 30,02 & 1,90 & 6,34 \\
\hline 1000 & 46,19 & 3,62 & 7,84 & 48,35 & 3,13 & 6,47 \\
\hline 2000 & 57,46 & 4,64 & 8,07 & 50,29 & 3,38 & 6,72 \\
\hline 4000 & 60,52 & 2,30 & 3,81 & 55,08 & 5,04 & 9,15 \\
\hline 6300 & 65,00 & 7,30 & 11,24 & 60,02 & 1,89 & 3,15 \\
\hline
\end{tabular}

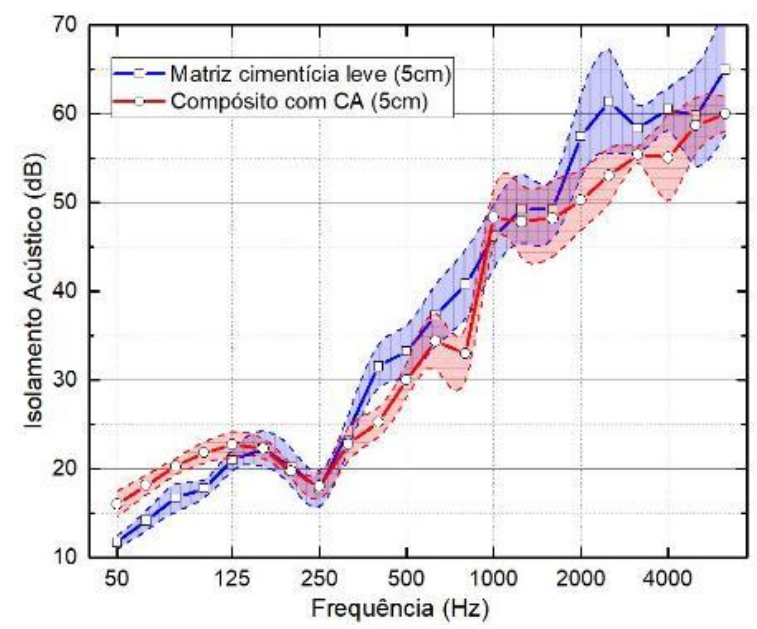

Figura 5: Curvas de isolamento acústico dos materiais

\subsection{Condutividade térmica}

Os valores referentes aos ensaios de condutividade térmica são apresentados pela Tabela 5. Foi considerada uma precisão de quatro casas decimais, uma vez que os valores sofrem alteração apenas a partir da segunda casa decimal. 
Tabela 5: Condutividade térmica.

\begin{tabular}{c|c|c|c}
\hline MATERIAL & $\begin{array}{c}\text { CONDUTIVIDADE TÉRMICA } \\
(\mathbf{W} / \mathbf{m} \cdot \mathbf{K})\end{array}$ & $\begin{array}{c}\text { D.P. } \\
(\mathbf{W} / \mathbf{m} . \mathbf{K})\end{array}$ & $\begin{array}{c}\text { C. V. } \\
(\%)\end{array}$ \\
\hline Matriz cimentícia leve & 0,2420 & 0,0097 & 4,00 \\
\hline Compósito com casca de arroz & 0,2756 & 0,0100 & 4,77 \\
\hline
\end{tabular}

De acordo com a Figura 6, observa-se que o compósito contendo casca de arroz apresentou maior condutividade térmica. Além disso, observa-se que as barras de dispersão não se sobrepuseram, indicando, portanto, uma diferença estatisticamente significativa entre os mesmos.

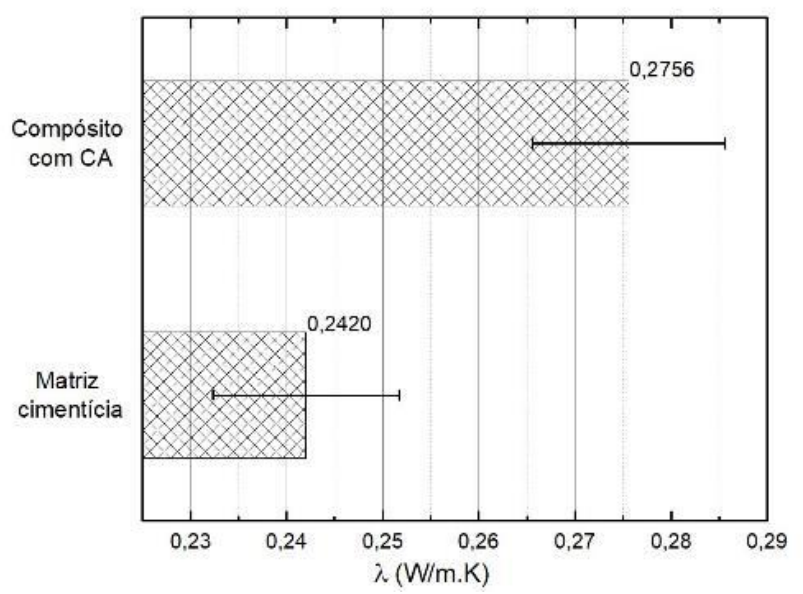

Figura 6: Condutividade térmica dos materiais.

Mesmo com a adição da casca de arroz as densidades da matriz e do compósito foram estatisticamente semelhantes. Entretanto, há consenso entre alguns autores $[9,11]$ de que materiais de mesma densidade podem apresentar valores distintos de condutividade térmica em decorrência da porosidade. Nesses estudos, o volume de fibra adicionada foi o fator principal para a redução da condutividade térmica, apontando uma relação inversamente proporcional. Nessa perspectiva [34, 35] estudaram os efeitos da adição de casca de arroz em materiais cimentícios e relataram o aumento da porosidade, o que a princípio deveria reduzir a condutividade térmica, e contraria os resultados encontrados.

Para o estudo realizado nesse trabalho a adição de casca de arroz à matriz não proporcionou a redução da condutividade térmica ao produto final. Assim, sugere-se que para a idade em que foram realizados os ensaios, as fibras vegetais estavam com sua estrutura, chamada de lumens, parcialmente saturada [36, 37], uma vez que possui matéria orgânica em sua composição, o que propícia à retenção de água [18]. Em vista disso, fica a perspectiva de que em idades mais avançadas essa água vai reagir com óxido de cálcio $(\mathrm{CaO})$ presente no cimento Portland, formando silicato de cálcio hidratado e hidróxido de cálcio $\left(\mathrm{Ca}(\mathrm{OH})_{2}\right)$. Assim, a sílica $\left(\mathrm{SiO}_{2}\right)$ presente na casca de arroz e o hidróxido de cálcio gerariam a reação pozolânica $\left(\mathrm{CSH}\right.$ ou $\left.\mathrm{CaO} \cdot \mathrm{SiO}_{2} \cdot \mathrm{H}_{2} \mathrm{O}\right)[27,28]$, sendo que esta reação será responsável por reduzir a quantidade de água e o volume ocupado pela matéria orgânica da $\mathrm{CA}$, e, como consequência, reduzir a condutividade térmica a um valor semelhante ou até mesmo inferior ao obtido pela matriz cimentícia [28].

\section{CONCLUSÕES}

No presente estudo foi avaliada a incorporação da casca de arroz à uma matriz cimentícia leve com o intuito de desenvolver um produto com bom desempenho térmico-acústico-mecânico para servir como alternativa aos materiais comumente utilizados para vedação. Além disso, oferecer um destino sustentável às fibras que atualmente são resíduos sem aplicações adequadas e que na grande maioria das vezes são queimadas para produção de energia elétrica e secagem de grãos [13].

A resistência mecânica à compressão axial do compósito, embora inferior à da matriz, atendeu ao mínimo requisitado pela NBR 13.438 [23] e pela NBR 15.270 [24]. O valor ficou inferior ao requisitado pela NBR 6136 [25], o que limita o uso do compósito para fins de vedação. 
De maneira coincidente à literatura [29, 30, 31, 32], o aumento da dimensão dos poros gerados pela adição da casca de arroz acarretou em uma melhoria significativa na absorção acústica do produto. Por outro lado, os resultados de isolamento acústico não foram estatisticamente diferentes aos resultados da matriz, também coincidente à literatura [30, 31, 33].

Quanto à condutividade térmica, a relação observada não seguiu a bibliografia e o compósito apresentou uma redução em seu desempenho em comparação à matriz. Entretanto, esse fato foi explicado em razão de uma relação condicionante ao produto: a retenção de água pelas fibras e as idades de ensaio. Visto que os ensaios foram realizados dos 28 aos 30 dias de cura, a matéria orgânica das fibras, que corresponde à $82,70 \%$ de sua composição [18], provavelmente reteve água, o que acabou preenchendo os vazios que seriam responsáveis por gerar maior isolação térmica. Por isso, existe a expectativa de que essa água seja absorvida pela hidratação do cimento e a condutividade térmica seja reduzida.

\section{AGRADECIMENTOS}

Os autores agradecem à CAPES pelo amparo financeiro para a realização da pesquisa e à FAPERGS pela concessão da bolsa de iniciação científica. Também agradecem à empresa ECOPORE pela doação do aditivo utilizado na pesquisa.

\section{BIBLIOGRAFIA}

[1] G1, Globo. Perícia Confirma que Espuma da Kiss Liberou Cianeto Durante Incêndio. Página da Internet. Disponível em: http://g1.globo.com/rs/rio-grande-do-sul/noticia/2013/03/pericia-confirma-queespuma-da-kiss-liberou-cianeto-durante-incendio.html. Acesso em 01 de julho de 2017, 19:09.

[2] UOL Notícias. Gás Tóxico Liberado na Queima de Espuma é a Causa de Mortes na Boate Kiss. Página da Internet. Disponível em: https://noticias.uol.com.br/cotidiano/ultimas-noticias/2015/09/25/gastoxico-liberado-na-queima-de-espuma-e-a-causa-de-mortes-na-boate-kiss.htm. Acesso em 17 de dezembro de 2017, 18:22.

[3] ASSOCIACAO BRASILEIRA DE NORMAS TECNICAS, NBR 15.575, Edificações habitacionais Desempenho, Requisitos gerais, Rio de Janeiro, 2013.

[4] CHIKHI, M., AGOUDJIL, B., HADDADI, M., et al., "Numerical modelling of the effective thermal conductivity of heterogeneous materials", Journal of Thermoplastic Composite Material, v. 26, pp. 336345, October, 2011.

[5] RAMAMURTHY, K., KUNHANANDAN NAMBIAR E.K., INDU SIVA RANJANI, G., "A Classification of Studies on Properties of Foam Concrete", Journal Cement \& Concrete Composites, v. 31, pp. 388-396, July, 2009.

[6] TEIXEIRA FILHO, F. J., TEZUCA, Y., Considerações sobre algumas propriedades dos concretos celulares espumosos, Boletim Técnico da Escola Politécnica da USP, São Paulo, 1992.

[7] MELO, G.F., Concreto celular polimérico: Influência da adição de resíduo de poliéster insaturado termofixo, Tese de D.Sc., Universidade Federal do Rio Grande do Norte, Natal, 2009.

[8] CANAHUA, S. F. C., "Los benefícios del concreto celular”, Revista Civilizate, v.3, n. 5, pp. 36-37, 2014.

[9] TONOLI, G. H. D., SANTOS, S., RABI, J., et al., "Thermal performance of sisal fiber-cement roofing tiles for rural constructions”, Journal Scientia Agricola, v.68, n.1, pp. 1-7, May, 2011.

[10] LERTWATTANARUK, P. SUNTIJITTO, A., "Properties of natural fiber cement materials containing coconut coir and oil palm fibers for residential building applications", Journal Construction and Building Materials, v. 94, pp. 664-669, September, 2015.

[11] KASHANI, A., NGO, T. D., MENDIS, P., et al., “A sustainable application of recycled tyre crumbs as insulator in lightweight cellular concrete", Journal of Cleaner Production, v. 149, pp. 925-935, April, 2017.

[12] INSTITUITO RIO GRANDENSE DE ARROZ. Página da internet. Disponível em: http://www.irga.rs.gov.br/conteudo/6911/safras. Acesso em julho de 2017.

[13] DELLA, V.P., KUHN, I., HOTZA, D., "Reciclagem de resíduos agroindustriais: cinza de casca de arroz como fonte alternativa de sílica”, Revista Cerâmica Industrial, v.10, pp.22-25, Abril, 2005.

[14] BENMANSOUR, N., AGOUDJIL, B., GHERABLI, A., et al., "Thermal and mechanical 
performance of natural mortar reinforced with date palm fibers for use as insulating materials in building", Journal Energy and Buildings, v. 81, pp. 98-104, May, 2014.

[15] FERNANDES, I. J., et al., "Caracterização do resíduo industrial casca de arroz com vistas a sua utilização como biomassa", In: $6^{\circ}$ Fórum Internacional de Resíduos Sólidos, São José dos Campos, São Paulo, Brasil, Julho, 2015.

[16] OLIVEIRA C. M., TANNOUS K. "Fluidodinâmica de biomassa em leito fluidizado gasoso", VIII Congresso Brasileiro de Engenharia Química em Iniciação Científica, 5f, Uberlândia, Minas Gerais, Brasil, 2009.

[17] MAYER, F.D. Aproveitamento da casca de arroz em uma micro central termoelétrica - Avaliação dos impactos econômicos e ambientais para o setor arrozeiro do Rio Grande do Sul, Dissertação M.Sc., Universidade Federal de Santa Maria, Santa Maria, 2009.

[18] FERREIRA, C.S. Desenvolvimento do processo de obtenção de filme polimérico a partir de cinza de casca de arroz, Dissertação M.Sc., Universidade Federal de Santa Catarina (UFSC), Florianópolis, 2005.

[19] INTERNACIONAL ORGANIZATION FOR STARDARDIZATION, ISO 10534-2, Acoustics Determination of sound absorption coefficient and impedance in impedance tubes, Part 2- Transferfunction method, 1996.

[20] ASSOCIAÇÃO BRASILEIRA DE NORMAS TÉCNICAS, NBR 5738, Concreto - Procedimento para moldagem e cura de corpos de prova, Rio de Janeiro, 2013.

[21] INTERNACIONAL ORGANIZATION FOR STARDARDIZATION, ISO 10534-1, Acoustics Determination of sound absorption coefficient and impedance in impedance tubes, Part 1- Method using standing wave ratio, 1996.

[22] AMERICAN SOCIETY FOR TESTING AND MATERIALS, ASTM C518, Standard Test Method for Steady-State Thermal Transmission Properties by Means of the Heat Flow Meter Apparatus, 2015.

[23] ASSOCIAÇÃO BRASILEIRA DE NORMAS TÉCNICAS, NBR 13.438, Blocos de concreto celular autoclavado - Requisitos, Rio de Janeiro, 2013.

[24] ASSOCIAÇÃO BRASILEIRA DE NORMAS TÉCNICAS, NBR 15.270, Componentes e requisitos, Parte 1- Blocos cerâmicos para alvenaria de vedação, Terminologia e requisitos, Rio de Janeiro, 2005.

[25] ASSOCIAÇÃO BRASILEIRA DE NORMAS TÉCNICAS, NBR 6136, Blocos vazados de concreto simples para alvenaria, Rio de Janeiro, 2014.

[26] JAUBERTHIE, R., RENDELL, F., TAMBA, S., et al., "Origin of the pozzolanic effect of rice husks", Journal Construction and Building Materials, v.14, pp. 419-423, December, 2000.

[27] JAUBERTHIE, R., RENDELL, F., TAMBA, S., et al., "Properties of cement - rice husk mixture", Journal Construction and Building Materials, v. 17, pp. 239-243, February, 2003.

[28] PACHLA, E.C., Desempenho térmico-acústico-mecânico-durabilidade de compósitos de matriz cimentícia com reduzida massa específica reforçados por casca e palha do arroz, Dissertação de M.Sc., Universidade Federal do Pampa, Alegrete - RS, 2017.

[29] PISELlO, A. L., FABIANI, C., MAKAREMI, N., et al., "Sustentainable New Brick and ThermoAcoustic Insulation Panel from Mineralization of Stranded Driftwood Residues", Journal Energies, v. 9, pp. 619, August, 2016.

[30] CORREDOR-BEDOYA, A.C., ZOPPI, R.A., SERPA, A. L. "Composites of scrap tire rubber particles and adhesive mortar - Noise insulation potential", Journal Cement \& Concrete Composites, v.82, pp.45-66, July, 2017.

[31] KIM, H.K., JEON, J.H., LEE, H.K., "Workability, and mechanical, acoustic and thermal properties of lightweight aggregate concrete with a high volume of entrained air", Journal Construction and Building Materials, v. 29, pp.193-200, November, 2011.

[32] LIMA, P. G., Absorção Sonora de Materiais Revestidos, Dissertação de M.Sc., Universidade Federal do Rio de Janeiro, Rio de Janeiro, 1999.

[33] GARCIA, D., Análise do Isolamento Sonoro de Sistemas Construtivos Residenciais Estruturados em Aço, Tese de D.Sc., Universidade Federal de Minas Gerais, Belo Horizonte, 2004.

[34] AHMAD, M. M., AHMAD, F., AZMI, M., et al., "Properties of Cement Mortar Consisting Raw Rice Husk", Journal Applied Mechanics and Materials, v. 802, pp.267-271, October 2015. 
[35] JANBUAlA, S., WASANAPIARNPONG, T., "Effect of Rice Husk and Rice Husk Ash on Properties of Lightweight Clay Bricks", Journal Key Enginneering Materials, v. 659, pp. 74-79, August, 2015.

[36] SILVA, F. A., CHAWLA, N., TOLEDO FILHO, R. D., "Tensile Behavior of High Performance Natural (Sisal) Fibers", Journal Composites Science and Technology, v. 68, pp. 3438-3443, December 2008.

[37] SAULO, S. F., SILVA, F. A., LIMA, P. R. L., et al., "Effect of hornification on the structure, tensile behavior and fiber matrix bond of sisal, jute and curauá fiber cement based composite systems", Journal Construction and Building Materials, v. 139, pp. 551-561, May, 2017.

\section{ORCID}

Eduardo Pachla https://orcid.org/0000-0003-0263-1562

Débora Bretas https://orcid.org/0000-0003-1477-0746

Wang Chong https://orcid.org/ 0000-0001-9486-7745

Ederli Marangon https://orcid.org/0000-0003-0812-8095

Pedro Jucá ～https://orcid.org/0000-0001-6588-3973 